\title{
Ein stabiles Olympisches Programm unter ständigem Modernisierungsdruck
}

\author{
Volker Kluge
}

\section{Abstract}

Im Mittelpunkt der Olympischen Spiele stehen die sportlichen Wettkämpfe, die vom IOC als Olympisches Programm bezeichnet werden. Über seine Komposition entscheidet die IOC-Session auf Vorschlag des Exekutivkomitees in Abstimmung mit dem Organisationskomitee der jeweiligen Spiele und den relevanten Internationalen Sportföderationen (IFs) - 28 für die Sommer-, sieben für die Winterspiele. Voraussetzung für die Austragung einer Sportart ist deren Übereinstimmung mit der Olympischen Charta und dem Welt-Antidoping-Code.

Das Programm, das aus Sportarten, Disziplinen und Wettkämpfen besteht, ist historisch gewachsen. Nachdem auf dem Olympischen Gründungskongress von 1894 eine erste Liste „wünschenswerter Sportarten" entstanden war, bestimmten kurzzeitig die Manager der Weltausstellungen von 1900 und 1904, in deren Rahmen die Spiele stattfanden, die Zusammensetzung. Erst danach war das IOC bedeutend genug, seine eigenen Vorstellungen zu realisieren.

Seitdem ist das Programm kontinuierlich gewachsen. Die Zahl der Disziplinen wuchs seit den ersten Spielen um rund das Siebenfache und die der Teilnehmer*innen auf mehr als das 40-fache. Trotz der enormen Ausweitung und der Veränderungen verfügt das Olympische Programm über eine große Stabilität. Dafür spricht auch die Tatsache, dass fünf Sportarten (Leichtathletik, Fechten, Radsport, Turnen und Schwimmen) bei allen Sommer- und vier (Nordischer Skisport, Eiskunstlauf, Eisschnelllauf und Eishockey) bei allen Winterspielen ausgetragen wurden.

Damit die Durchführung der Olympischen Spiele organisatorisch und finanziell langfristig beherrschbar bleibt und das Interesse der Zuschauer nicht erlahmt, unterliegt das IOC einem ständigen Zwang zur Modernisierung. Im Laufe der Jahrzehnte gab es zahlreiche Reformen, um die Interessen der verschiedenen Stakeholder angemessen zu berücksichtigen. Jüngster Ausdruck ist die Olympische Agenda 2020 und ihre Fortsetzung 
als Agenda 20+5, die auf die drei Säulen Glaubwürdigkeit, Nachhaltigkeit und Jugend setzt.

\section{Einleitung}

Am 25. November 1892 sah der damals 29-jährige Pierre de Coubertin die Stunde gekommen, eine Rolle im Sport auf internationaler Ebene zu übernehmen. Die Gelegenheit bot sich in Paris anlässlich der Fünf-JahrFeier der Vereinigung der französischen Sportverbände (USFSA) ${ }^{1}$, die mit einer Konferenz in der Sorbonne begangen wurde.

Nach Vorträgen über Leibesübungen im Altertum und Mittelalter widmete sich Coubertin dem Sport in der modernen Welt. Seine Rede beendete er mit den Worten:

„Lassen Sie uns Ruderer, Läufer, Fechter ins Ausland senden; das ist das Freihandelssystem der Zukunft [...] und der Sache des Friedens wird eine neue und mächtige Stütze erwachsen. Das genügt, dass ich [...] nunmehr an den zweiten Teil des Vorhabens denken kann [...]: die Wiedererweckung der Olympischen Spiele“ (Müller, 1981, S. 22).

Was Coubertin für eine sensationelle Neuigkeit gehalten hatte, blieb in Wirklichkeit ohne Resonanz. Das Auditorium applaudierte brav, doch kaum einer war in der Lage, die Tragweite zu erfassen und Coubertins Idee von den Formen der Antike zu trennen. Beim Verlassen des Saales fragten einige, was er denn mit „Wiedererweckung“ gemeint hätte. Sie verstanden den Begriff lediglich symbolisch. Man konnte sich ein solches Projekt vielleicht als Theaterstück vorstellen, nicht aber in der Realität. Andere witzelten und sie erkundigten sich, ob Frauen zugelassen würden und allgemeine Nacktheit Pflicht wäre (Coubertin, 1997).

Das nächste Frühjahr kam, ohne dass Coubertins Vorschlag die Öffentlichkeit erreicht hatte. Da nichts passierte, griff er zu einem Trick. Er erinnerte sich, dass der Herausgeber der Zeitschrift Les Sports Atblétiques, Adolphe de Pallisaux, vor einiger Zeit die Einberufung eines internationalen Kongresses vorgeschlagen hatte, um auf ihm die Amateurregeln zu diskutieren, eine Idee, die man damals zu den Akten gelegt hatte. Gemeinsam gruben sie das Papier wieder aus, und dieses Mal - am 1. August 1893 - stimmte das USFSA-Präsidium zu, einen Kongress zum

1 Union des Sociétés françaises des Sports athlétiques. Coubertin übte darin die Funktion des Generalsekretärs aus. 
Studium der Amateurprinzipien und zur Erneuerung der Olympischen Spiele ${ }^{2}$ auszurichten. Als Termin wurde Juni 1894 festgelegt.

Um eine möglichst große öffentliche Aufmerksamkeit zu erhalten, konzentrierte Coubertin seine Energie auf die festliche Eröffnung am 16. Juni 1894, die im Großen Amphitheater der Sorbonne stattfand und an der etwa 2000 Zuhörer - darunter zahlreiche Student*innen und Schüler*innen - teilnahmen. Von den 49 aufgeführten Vereinigungen und Clubs waren in Wirklichkeit nur 37 anwesend. Die Zahl der Delegierten belief sich auf 78. Mehr als zwei Drittel waren Franzosen (IOC, 1894a). ${ }^{3}$ Die ausländischen Abordnungen beschränkten sich auf 20, die acht Länder repräsentierten. Deutschland war nicht vertreten.

Als zwei Tage später die eigentlichen Beratungen begannen, waren die Reihen bereits gelichtet. Die Verbliebenen - lediglich 42 - hatten die Wahl zwischen zwei Kommissionen. In der ersten wurde die Amateurproblematik beraten, während die zweite, für die sich anfangs nur 17 Delegierte interessierten, sich mit der Möglichkeit der Wiedereinführung der Olympischen Spiele beschäftigte. Außerdem wollte Coubertin zwei weitere Punkte klären lassen. Sie betrafen Fragen der Organisation und der Nominierung eines Leitungsgremiums, des Internationalen Olympischen Komitees (IOC) (Kluge, 2019).

Der in Paris lebende griechische Literat Dimitrios Vikelas, den Coubertin mit der Präsidentschaft der olympischen Kommission beauftragt hatte, verlas zur Eröffnung ein Memorandum über die antiken Spiele, in dem das Pentathlon als Ideal gepriesen wurde (IOC, 1894b). Auch die beiden schwedischen Delegierten legten Wert auf die Austragung des Pentathlons.

Coubertin, der zwischen den Kommissionen hin- und herpendelte, gab jedoch zu bedenken, dass inzwischen auch Sportarten wie Rudern entstanden waren, die die alten Griechen nicht kannten. Offenbar dauerte es einige Zeit, bis die Delegierten begriffen hatten, dass es Coubertin um modernen Sport ging und dass er die Wettkämpfe lediglich unter ein schützendes Dach stellen wollte, das „ihnen den Nimbus der Größe und des Ruhmes verleihen könnte: unter den Schutz des klassischen Altertums" (Coubertin et al., 1897, S. 5).

2 Congrès international de Paris pour l'étude et la propagation des principes d'amateurisme.

3 Coubertin operierte mit der Zahl 79, siehe: Revue Olympique, Nr. 1, Januar 1901, S. 7. 
In seinen Memoiren schrieb Coubertin, dass man seine Vorschläge ohne Widerspruch akzeptiert hätte (Coubertin, 1997). Das Protokoll sagt etwas anderes aus. Es wurde lebhaft diskutiert, und der "Chef" konnte sich keineswegs immer mit seinen Vorstellungen durchsetzen. So galt nicht etwa Paris, das Coubertin für 1900 als Schauplatz der Premiere vorgesehen hatte, als Favorit, sondern London. Außerdem meinten einige, dass der Zeitraum von sechs Jahren bis zur erstmaligen Austragung zu lang wäre, worauf Coubertin in der nächsten Sitzung selbst zur Überraschung von Vikelas Athen 1896 ins Gespräch brachte (IOC, 1894c). ${ }^{4}$ Einigkeit herrschte indes, dass die Spiele im Vierjahresrhythmus stattfinden und bei ihnen mit Ausnahme des Fechtens nur Amateure an den Start gehen sollten.

Bei den Sportarten war unstrittig, dass die Leichtathletik - mit dem Pentathlon als Krönung - das Kernstück der Spiele darstellen sollte. Die Aufnahme des Wassersports, Schwimmen, Rudern und Segeln, war ebenso selbstverständlich wie die der Kampfsportarten (Fechten, Boxen, Ringen), Reiten, Radsport und Turnen sowie der Sportspiele (Fußball, Tennis, Paume etc.). Den Alpinisten wurde ein Preis für den „interessantesten Aufstieg" in Aussicht gestellt (IOC, 1894a).

Auf Wunsch der Schweden gelangte auch „patinage“ (Eislauf) auf die Liste, ohne die dafür notwendigen Bedingungen erörtert zu haben (IOC, 1894a). Abgelehnt wurde der Antrag, Wettkämpfe für Jugendliche auszuschreiben - ein Vorschlag, den zuvor Vikelas mit Verweis auf die Antike angeregt hatte. Anders als in der Vergangenheit sollten die modernen Spiele ausschließlich erwachsenen Männern vorbehalten bleiben. Die Teilnahme von Frauen stand nicht zur Debatte.

Das erste offizielle Wettkampfprogramm für die Spiele der I. Olympiade trägt das Datum vom 12. bis 24. November 1894, aufgestellt vom Organisationskomitee, das sich an diesem Tage in Athen unter Vorsitz des griechischen Kronprinzen und in Anwesenheit von Coubertin, der für zwei Jahre die Funktion des IOC-Generalsekretärs übernommen hatte, konstituierte. Das Programm, datiert sowohl nach dem in Griechen-

4 Leider ist das Protokoll vom ersten Sitzungstag (18. Juni) nicht vorhanden. In „Einundzwanzig Jahre Sportkampagne (1887-1908)“, Henn Verlag, Ratingen 1974, S. 80-81, schreibt Coubertin, dass er den Vorschlag, die ersten Spiele schon 1896 in Athen auszutragen, gemeinsam mit Vikelas eingebracht hätte. Im Brief von Vikelas (19. Juni 1894) an Coubertin heißt es jedoch: „Leider habe ich Sie nach unserer Sitzung nicht mehr gesehen. Ich wollte Ihnen sagen, wie sehr ich mich über Ihren Vorschlag gefreut habe, in Athen den Anfang zu machen. Ich bedaure nur, dass es mir nicht möglich war, ihn besser zu unterstützen.“ 
land gültigen Julianischen als auch nach dem Gregorianischen Kalender, wurde unterteilt in sieben Sportzweige: a) Athletischer Sport (Leichtathletik); b) Turnen (Gerätturnen, Gewichtheben); c) Fechten und Ringen; d) Schießen; e) Nautischer Sport (Segeln, Rudern, Schwimmen); f) Radfahren; g) Athletische Spiele (Tennis, Kricket, Fußball) (Mallon \& Widlund, 1998).

Bald darauf wurde diese Liste aber modifiziert. Zum Marathonlauf, den der deutsch-französische Philologe Michel Bréal angeregt und für den er einen Siegerpokal gestiftet hatte, kam ein Marathon-Radrennen über 87 km. Hingegen wurden Fußball (unterteilt in Rugby und „Association"), Reiten, Segeln und Kricket mangels Anmeldungen gestrichen. Ausgetragen wurden schließlich 43 Disziplinen in neun Sportarten, wobei manches aus heutiger Sicht kurios erscheint: so das griechisch-römische Ringen und das Gewichtheben, jeweils in nur einer Kategorie; ein 100-m-Schwimmen für Matrosen der griechischen Kriegsmarine; ein 24Stunden-Radrennen auf der Bahn oder das Tauhangeln als Bestandteil der Turnwettkämpfe. Auch Rudern als zehnte Sportart gehörte zum Programm. Doch die Regatta musste wegen der stürmischen See abgesagt werden.

\section{Der Fehler, Olympische Spiele an Weltausstellungen anzudocken}

Coubertin, der nach Athen das Amt des IOC-Präsidenten übernommen hatte ${ }^{5}$, wollte aus „Budget-Gründen“ die nächsten Spiele an die Pariser Weltausstellung ankoppeln. Doch schon sein erstes Gespräch mit dem Generalbevollmächtigten, Alfred Picard, endete enttäuschend. Auch bei Frankreichs Präsident Félix Faure erreichte er nichts. Er entschloss sich

5 Auf dem Olympischen Kongress 1894 hatte man vereinbart, dass der IOC-Präsident für die Dauer einer Olympiade jeweils ein Vertreter des Gastgeberlandes der nächsten Olympischen Spiele sein sollte. Da nach Paris die nächsten Spiele 1904 in die USA vergeben wurden, wäre turnusgemäß der US-Amerikaner William M. Sloane an der Reihe gewesen, der sich aber außerstande sah, das Amt auszuüben. Stattdessen schlug er 1901 vor, Coubertin die Präsidentschaft lebenslang zu übertragen. Dieser lehnte ab, er war aber bereit, das IOC für weitere zehn Jahre zu leiten. Nach den sogenannten Olympischen Zwischenspielen von 1906 in Athen, die gegen seinen Willen stattgefunden hatten und nach denen er bemüht war, das verlorengegangene Vertrauen der Mitglieder zurückzugewinnen, stellte er sich 1907 einer Abstimmung ohne Gegenkandidat, in der er erneut für ein Jahrzehnt gewählt wurde. Coubertin Amtszeit endete aber auch nicht 1917, sondern erst 1925 - aus ursprünglich vier waren 28 Jahre geworden. 
deshalb, die Vorbereitung in die Hände eines privaten Komitees unter Leitung eines Diplomaten, des Vicomte Charles de La Rochefoucauld, zu legen, von dessen Finanzkraft er sich einiges versprach.

Im Jahr 1898 konstituierte sich das 40-köpfige „Comité La Rochefoucauld“, das bei den Vorbereitungen auf die Unterstützung der französischen Sportverbände angewiesen war. Dabei kam es zu einer Vielzahl von Kompetenzrangeleien. Am 9. November 1898 brach die USFSA die Beziehungen ab.

Schwierigkeiten machte auch die Leitung der Weltausstellung. Als das Generalsekretariat im Februar 1899 ein eigenes Organisationskomitee einsetzte, trat zwei Monate später La Rochefoucauld zurück und sein Komitee erklärte die Selbstauflösung. Da blieb Coubertin nichts anderes übrig, als ebenfalls zu kapitulieren. Bei den Olympischen Spielen von 1900, die nur im Ausland so bezeichnet wurden, hatte das entmachtete IOC nichts zu sagen (Durry, 2019).

Anstelle des Standardprogramms von 1896, das Coubertin um Reiten, Bogenschießen, Eislauf und einige Spielsportarten erweitern wollte, wurden Weltausstellungswettkämpfe ausgerichtet, genannt "Concours nationaux et internationaux d'exercices physiques et de sports". Sie waren unterteilt in zehn internationale und zwei nationale Sektionen sowie eine Wissenschafts- und Hygiene-Abteilung. Die Wettkämpfe, zu denen sowohl Amateure und in einigen Sportarten auch Berufssportler zugelassen waren, begannen Mitte Mai, und einige endeten erst im Oktober.

Im Unterschied zu Athen bot Paris die gesamte Breite des Sommersports mit Ausnahme von Boxen und Ringen. Zum Programm gehörte auch eine Reihe von Sportspielen, Schlagballspiele sowie technische Sportarten wie Motor- und Flugsport, die manche Chronisten zu Unrecht als „nichtolympisch“ einstufen.

In der Geschichtsschreibung wird Paris 1900 bisweilen als „Chaos von 162 Tagen“ dargestellt, was mit der Wirklichkeit nicht übereinstimmt. Das Gegenteil war der Fall, allerdings waren es keine Olympischen Spiele nach den Vorstellungen Coubertins und seiner Mitstreiter. Die Wettkämpfe, zu denen auch Handicap-Konkurrenzen, aber keine Volksbelustigungen wie Sackhüpfen, Tonnenrollen und Kirschkernweitspucken gehörten, wie man in vielen Quellen lesen kann, passen nur schlecht in das Raster, das heutzutage an Olympische Spiele angelegt wird.

Ähnliches gibt es über die Spiele der III. Olympiade zu sagen, die 1901 vom IOC zuerst nach Chicago, dann aber auf Bitten von US-Präsident Theodore Roosevelt nach St. Louis verlegt wurden, das 1903 den 100. Jahrestag des Verkaufs von Louisiana („Louisiana Purchase“) durch Napoleon an die Vereinigten Staaten mit einer Weltausstellung begehen 
wollte. Da die Louisiana Purchase Exposition aber nicht rechtzeitig fertig wurde, verlegte man sie aufs nächste Jahr, womit die darin integrierten Sportwettkämpfe sogar noch in den olympischen Zyklus passten.

Geleitet wurde die Abteilung „Physical Culture“ von James E. Sullivan, dem mächtigen Generalsekretär der Amateur Athletic Union (AAU) ${ }^{6}$ und einem Gegenspieler Coubertins, der seinerseits auf eine Reise nach St. Louis verzichtete, weil dem IOC erneut keine Kompetenzen eingeräumt wurden. Er beauftragte den Deutschen Dr. Willibald Gebhardt und den Ungarn Ferenc Kemény, ihn als IOC-Delegierte zu vertreten.

Wie vier Jahre vorher bestand das auf sieben Monate verteilte Programm aus einer Vielzahl von internationalen und nationalen Wettbewerben, die von den Amerikanern teilweise als „olympisch“ oder als "Championships" verschiedener Kategorien bezeichnet wurden. Der Begriff „Olympic Games“ wurde nur für den Zeitraum vom 29. August bis 3. September 1904 verwendet, in dem 26 Wettkämpfe in der Leichtathletik einschließlich Tauziehen und Gewichtheben stattfanden (Sullivan, 1905).

6 Dachorganisation des US-amerikanischen Amateursports. 
Abbildung 1: Kopfweitsprung gehörte 1904 in St. Louis zu den Disziplinen des Schwimmsports. Gemessen wurde die zurückgelegte Strecke, wenn entweder das Gesicht des Wettkämpfers auf der Wasseroberfläche aufgetaucht bzw. das Zeitlimit von 60 Sekunden erreicht war. Auf dem Foto: Der US-Amerikaner Edgar H. Adams kam auf 17,53 m, womit er Platz zwei belegte.

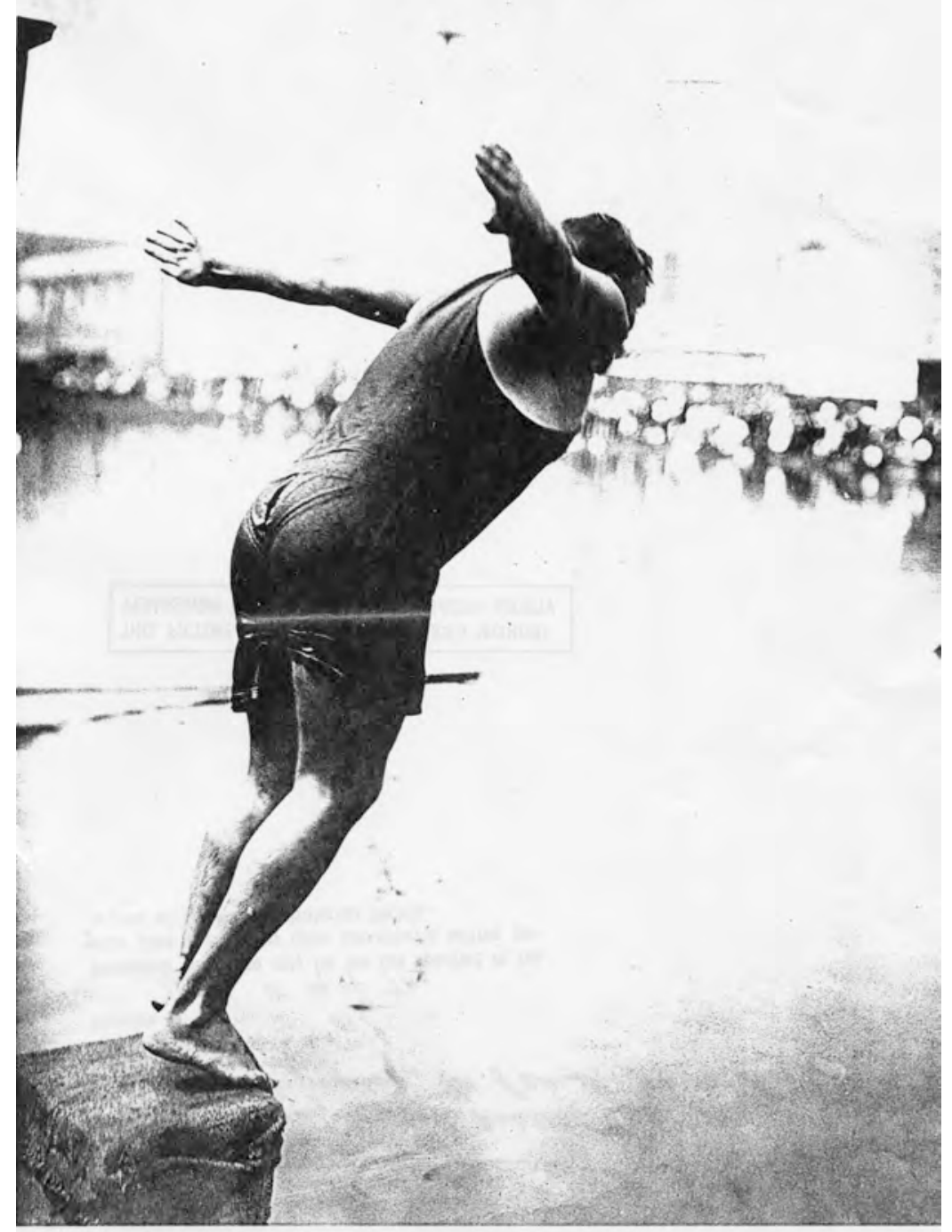

(Archiv Volker Kluge) 
Bei der Bewertung der übrigen Sportarten und Wettbewerbe verlässt sich das IOC in seinen Veröffentlichungen auf das Urteil von Historikern und damit auf die umstrittene subjektive Einordnung von „olympisch“ und „nichtolympisch“. Rückwirkend ist das auch schwierig, denn welche Maßstäbe sollen angelegt werden? Selbst eine internationale Beteiligung ist nur bedingt ein Kriterium, da in Sportarten wie Ringen, Boxen, Bogenschießen, Bahnradsport, Wasserball, Rudern, Basketball und Roque - eine Variante des Krockets - ausschließlich US-Athleten am Start waren.

Die wenigen Ausländer, die an diesen Spielen teilnahmen, kamen entweder aus Kanada oder Kuba, oder sie lebten oder arbeiteten vorübergehend in Übersee. Kleine Olympiateams nach heutigem Verständnis schickten nur Deutschland und Ungarn. Und die Gastgeber hatten sowieso ihre eigene Sicht auf die Dinge. Für sie wurde die Punktwertung vom New York AC knapp vor dem Chicago AA gewonnen. Da nur die Leichtathletik gezählt wurde, landeten die Deutschen hinter einer Reihe weiterer amerikanischer Clubs mit zwei Punkten nur auf dem viertletzten Platz (Lucas, 1905).

\section{Ein modernes Pentathlon und ein solches für die fünf Musen}

Coubertins Absicht, die Spiele von den Weltausstellungen zu trennen, ließ sich auch 1908 nicht völlig verwirklichen. Da das von ihm als Austragungsort vorgesehene Rom zwei Jahre vorher eine Absage erteilte, nahm er dankend das britische Angebot an, die Spiele im Rahmen der FrancoBritish Exhibition auszutragen, womit die Finanzierung gesichert war.

Was das Programm betraf, so orientierten sich die Gastgeber anfangs an dem der Pariser Weltausstellung von 1900, allerdings wurden später die geplanten Wettbewerbe im Golf, Flug- und Automobilsport - vorgesehen war auch ein „Flying Kilometre" für Rennwagen - gestrichen. Übrig blieben die Motorbootrennen, für die es jedoch kaum Meldungen gab.

Erstmals durfte auch das IOC mitreden, das auf seiner Session von 1907 in Den Haag die Teilung der Spiele in zwei Abschnitte bestätigt hatte. Für die „Summer Games“ und deren „Stadiondisziplinen“ ließ das Ausstellungskomitee eine Multifunktionsarena mit Lauf- und Radrennbahn, einem Schwimmbecken sowie Plätzen für Turnen und Ringkampf errichten. Irritiert zeigten sich die Briten jedoch von der Festlegung des IOC, das metrische System als offiziell anzuerkennen. Im Oktober folgten dann die „Winter Games" mit Boxen, den Spielsportarten Fußball, Feldhockey, Lacrosse und Rugby sowie erstmals auch Eiskunstlauf. 
Hingegen musste Coubertin seinen 1906 verkündeten Plan, einen „Fünfkampf der Musen“ auszuschreiben, auf Stockholm 1912 verschieben. Und auch dann war er im Wesentlichen auf sich allein gestellt, da die Schweden daran kein Interesse zeigten und sich außerstande erklärten, die Wettbewerbe in der Architektur, Literatur, Musik, Malerei und Bildhauerkunst objektiv zu bewerten. Anders verhielt es sich mit Coubertins zweitem Projekt, dem die Schweden sofort zustimmten. Der Moderne Fünfkampf mit den Disziplinen Pistolenschießen, Schwimmen, Fechten, Reiten und Geländelauf, war als Äquivalent zum antiken Vorbild gedacht.

Die Absicht der Schweden, die Wettkämpfe auf eine Dauer von zehn bis zwölf Tagen zu verkürzen, ließ sich nur teilweise realisieren. Es gab im Juli eine „Olympische Woche“, die als Kernstück galt. Die Spiele begannen jedoch schon im Mai/Juni mit Tennis, Fußball und Schießen. Da aus dem Programm von 1908 Bogenschießen, Boxen, Bahnradsport, Hockey, Lacrosse, Racquets und Freistilringen gestrichen wurden, reduzierte sich dieses von 21 auf 13 Sportarten. Neu und eine prachtvolle Bereicherung waren lediglich die Reiterspiele.

Abbildung 2: Sprünge aus dem Stand - Weit-, Drei- und Hochsprung - zählten zum Leichtathletik-Programm der frühen Olympischen Spiele. Einer der Nachfolger des US-Amerikaners Raymond Ewry, der von 1900 bis 1908 alle Wettbewerbe gewann, wurde der Grieche Konstantin Tsiklitiras. Er wurde 1912 in Stockholm Olympiasieger im Weitsprung aus dem Stand mit 3,37 m.

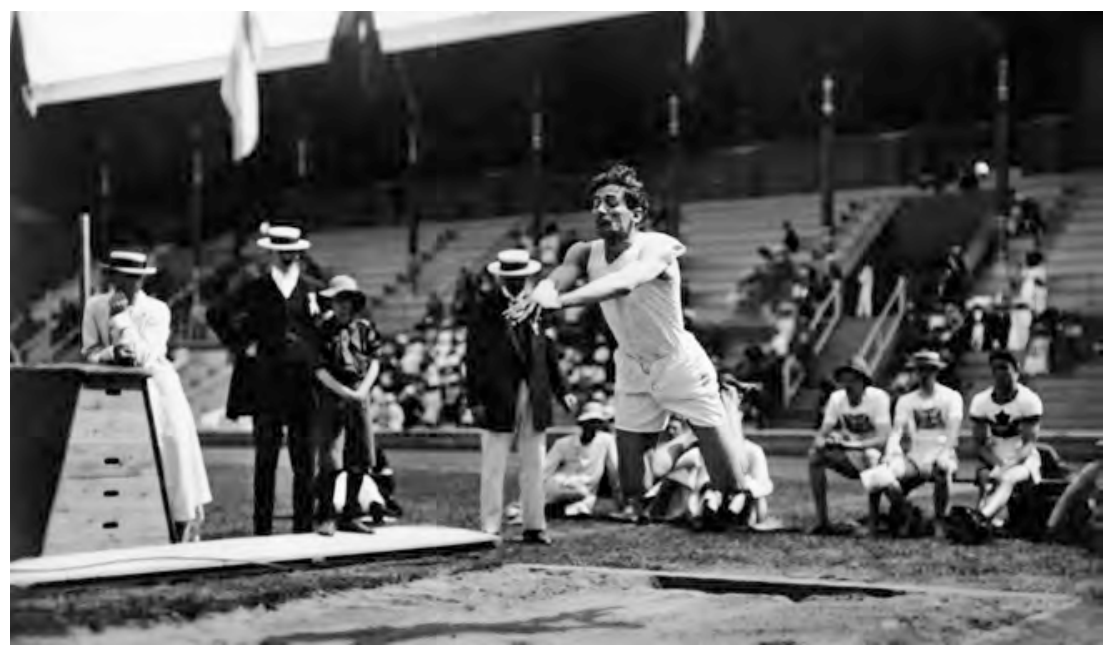

(Archiv Volker Kluge) 
Die Frauen, deren Teilnahme 1908 auf Bogenschießen, Tennis und Eiskunstlauf beschränkt war, wurden erstmals im Schwimmen zugelassen. Zum Ärger von Coubertin, der Frauensport für „unpraktisch, uninteressant, unbeholfen und, ich zögere nicht, das hinzuzufügen, unanständig“ hielt (Coubertin, 1912, S. 110-111). Sein olympisches Ideal blieb zeitlebens das „feierliche und periodische Auftreten der männlichen Athletik“ in einem „künstlerischen" Umfeld „mit dem Applaus der Frauen als Belohnung" (Coubertin, 1912, S. 110-111).

Aus anderen Gründen lehnte er den Vorschlag des italienischen IOCMitglieds Graf Eugenio Brunetta d'Usseaux ab, die für Januar 1913 geplanten Nordischen Spiele als Teil der Olympiade zu akzeptieren und später eigenständige Winterspiele einzuführen. In den alpinen Hoteliers sah Coubertin die "Strohmänner habgieriger Aktionäre“ und in ihrer Kundschaft „laute und überall störende Vergnügungsreisende“, mit denen der Tango und die gefärbten Haare in die Berge kämen (Coubertin, 1914, S. 39f). Das Generalsekretariat für die 1916 in Berlin geplanten Spiele war dennoch nicht davon abzuhalten, für den Februar jenes Jahres Wettkämpfe im Skisport auf dem Feldberg und in den drei Eissportarten im Berliner Eispalast anzusetzen. Mit Beginn des Ersten Weltkriegs hatten sich alle Entwürfe erledigt.

\section{Das gewachsene Selbstbewusstsein der internationalen Sportfachverbände}

Kurz vor Kriegsbeginn fand anlässlich der 20-Jahrfeier des IOC im Juni 1914 in Paris ein weiterer Olympischer Kongress statt, auf dem eine umfassende Programmdiskussion nicht mehr aufzuhalten war und an der auch Abgesandte der Nationalen Olympischen Komitees (NOKs) teilnahmen. Die Delegierten einigten sich auf eine Einteilung in obligatorische und fakultative Sportarten. Neu gegenüber Stockholm 1912 waren Golf, Polo, Rugby und Hockey sowie die Wintersportarten, die sämtlich als fakultativ galten (IOC, 1919).

$\mathrm{Zu}$ Meinungsverschiedenheiten führte die Absicht der Gastgeber, das Turnen nach der deutschen Wettordnung, d.h. mit einem Mehrkampf, zu dem „volkstümliche Übungen“ gehörten, auszurichten. Gegen den Widerstand der Organisatoren verlangte das IOC auch das in Deutschland noch verbotene Boxen.

Vier Jahre später war es ein Wunder, dass sich ausgerechnet Belgien, das unter dem Krieg und seinen katastrophalen Folgen am meisten zu leiden hatte, bereits 1920 in der Lage sah, in Antwerpen das nächste olympische Fest auszurichten, allerdings unter der Bedingung, die Kriegsverlierer - 
Deutschland und seine Verbündeten - auszuschließen. Verteilt auf fünf Monate, wurde ein Programm von 21 Sportarten einschließlich Eiskunstlauf und Eishockey auf die Beine gestellt. Lediglich Golf wurde mangels Interesses abgesagt.

Eine wichtige Unterstützung leisteten die 19 internationalen Sportfachverbände (IFs), die oftmals auch eigene Vorstellungen von einem zukünftigen Programm verfolgten, denen sich das IOC zu stellen hatte. Eigens deshalb wurde 1921 ein Kongress nach Lausanne einberufen, auf dem die IFs ihr gewachsenes Selbstbewusstsein zum Ausdruck brachten.

Die vom IOC erhoffte Straffung des Programms kam nicht zustande, eliminiert wurden lediglich Bogenschießen, Golf und - vorübergehend Feldhockey. Die Turner, die sich seit Längerem über das „richtige“ System gestritten hatten und 1920 unter drei verschiedenen Teamwettbewerben wählen konnten, einigten sich auf einen Mehrkampf von Pflicht- und Kürübungen an den Geräten. Die Unterteilung in obligatorische und fakultative Sportarten von 1914 wurde aufgehoben (IOC, 1921).

Der nachhaltigste Beschluss war jedoch das Zugeständnis an Paris 1924, als „Vorspiel“ in Chamonix eine „Internationale Wintersport-Woche“ zu veranstalten. ${ }^{7}$ Sie verlief dann so erfolgreich, dass auf dem nächsten Olympischen Kongress 1925 in Prag beschlossen wurde, die Wettkämpfe rückwirkend als „First Winter Olympic Games“ anzuerkennen und zukünftig für die Winterspiele einen eigenen Zyklus einzuführen (Czechoslovak Organising Committee, 1925).

Der Prager Kongress bestätigte auch das Programm der Sommerspiele, das vom IOC weiterhin in Sportzweige eingeteilt wurde: Leichtathletik, Turnen, Kampfsport (Boxen, Fechten, Ringen), Wassersport (Rudern, Schwimmen), Reitsport, kombinierter Sport (Moderner Fünfkampf), Fußball, Kunstwettbewerbe. Den Organisationskomitees war es freigestellt, die Palette um Radsport, Segeln (Monotype), Gewichtheben und weitere Spielsportarten zu ergänzen (Czechoslovak Organising Committee, 1925). ${ }^{8}$

Voraussetzung war die Einhaltung der Amateurbestimmungen, weshalb Fußball, Schießen und Tennis zur Disposition standen, da deren Weltver-

7 Im Programm standen die Sportarten nordischer Skisport, Eiskunstlauf, Eisschnelllauf, Bobsport, Militärpatrouillenlauf und Curling.

8 Den IFs war es freigestellt, die olympischen Wettkämpfe als Weltmeisterschaften zu werten. Im englischen Originaldokument wurde der Kampfsport als „SelfDefence Sports“ bezeichnet. 
bände die Definition der Regeln für sich allein beanspruchten. ${ }^{9}$ Letztlich zeigte sich die FIFA für Amsterdam 1928 in der Frage des „Verdienstausfalls"10 kompromissbereit. Dennoch verwirklichte sie anschließend ihre Drohung, im Juli 1930 in Uruguay die erste Weltmeisterschaft auszurichten, zu der auch Profis zugelassen waren. Daraufhin strich die IOCExekutive das Fußballturnier für die Spiele von Los Angeles 1932.

Ein anderer Streitpunkt war die Zulassung von Frauen, deren Emanzipation mit Kriegsende in allen Bereichen zugenommen hatte. Eine Vorkämpferin des Frauensports war die Französin Alice Milliat. Es war ihre Initiative, 1921 eine Dachorganisation namens Fédération Sportive Féminine Internationale (FSFI) mit dem Ziel zu gründen, alle vier Jahre "Jeux Olympiques Féminins", Olympische Spiele für Frauen, auszurichten. Die ersten, auf die Leichtathletik begrenzt, fanden 1922 in Paris statt.

Notgedrungen mussten sich IOC und Internationaler Leichtathletikverband (IAAF), die den Erfolg der FSFI besorgniserregend fanden, mit der neuen Konkurrenz beschäftigen. Die IAAF erklärte sich bereit, die Zuständigkeit für den Frauensport in ihre Statuten aufzunehmen und 1928 in Amsterdam erstmals fünf Disziplinen auszuschreiben, doch auch danach hielt das Tauziehen an. Die von dem schwedischen IAAFPräsident Sigfrid Edström geleitete Programmkommission schlug dem Olympischen Kongress von 1930 in Berlin mit 26:1-Stimmen vor, die weibliche Teilnahme auf Turnen, Schwimmen, Tennis und Eiskunstlauf einzuschränken (IOC, 1930).

Nach heftigen Diskussionen gelang es dann im zweiten Anlauf, sich auch noch auf die Leichtathletik (17:9) und das Fechten (19:8) zu einigen. Die FSFI blieb aber weiterhin selbständig. Erst 1936 gab es eine Annäherung zur IAAF, so dass seitdem gemeinsame internationale Meisterschaften möglich wurden.

Immerhin gelang es auf diesem Kongress, die Sommerspiele einschließlich Eröffnung auf 16 Tage zu verkürzen (IOC, 1930). Die Dauer der Winterspiele legte man nicht fest. Sie wurden erst später auf zehn Tage begrenzt, vorausgesetzt, dass sie ohne Wetterkapriolen über die Bühne gehen konnten (IOC, 1955b).

9 Der Tennis-Weltverband ILTF (später ITF) verbot 1926 seinen Mitgliedsverbänden die Teilnahme in Amsterdam, da das IOC nicht bereit war, der Reamateurisierung von Berufsspielern zuzustimmen. Daraufhin verschwand Tennis bis zu seiner Wiederaufnahme von 1987 aus dem Olympischen Programm.

10 Vergütungen, die an Spieler oftmals in verschleierter Form als Entschädigung für Verdienstausfall gezahlt wurden. Nach den Festlegungen des Prager Kongresses verstießen diese gegen die Amateurregeln. 
Das Programm von Los Angeles mit 14 Sportarten und 117 Disziplinen war überschaubar. Hinzu kamen noch Kunstwettbewerbe und zwei Demonstrationssportarten nach Wahl des Gastgebers (IOC, 1921). Aus Prestigegründen verlangte Hitler, der sich mit den Spielen von Berlin 1936 als „Friedenskanzler“ darstellen wollte, vier Jahre später alles Bisherige zu übertreffen. Das galt auch für das Wettkampfprogramm, das mit 19 Sportarten und 129 Disziplinen noch einmal deutlich aufgestockt wurde. ${ }^{11}$ Die Auswahl wurde nicht dem Zufall überlassen. Mit Ausnahme von Basketball und Polo orientierte sich das Organisationskomitee mit seinen Vorschlägen vorrangig an deutschen Medaillenchancen.

Abbildung 3: Das Polospiel, bei dem zwei Mannschaften mit jeweils vier Reitern versuchen, mit Schlägern einen kleinen Ball ins gegnerische Tor zu treiben, stand ab Paris 1900 fünfmal im Olympischen Programm. Letztmals wurde es 1936 bei den Spielen in Berlin ausgetragen. Sieger wurde das argentinische Team (in Weiß), das Großbritannien mit 11:0 besiegte.

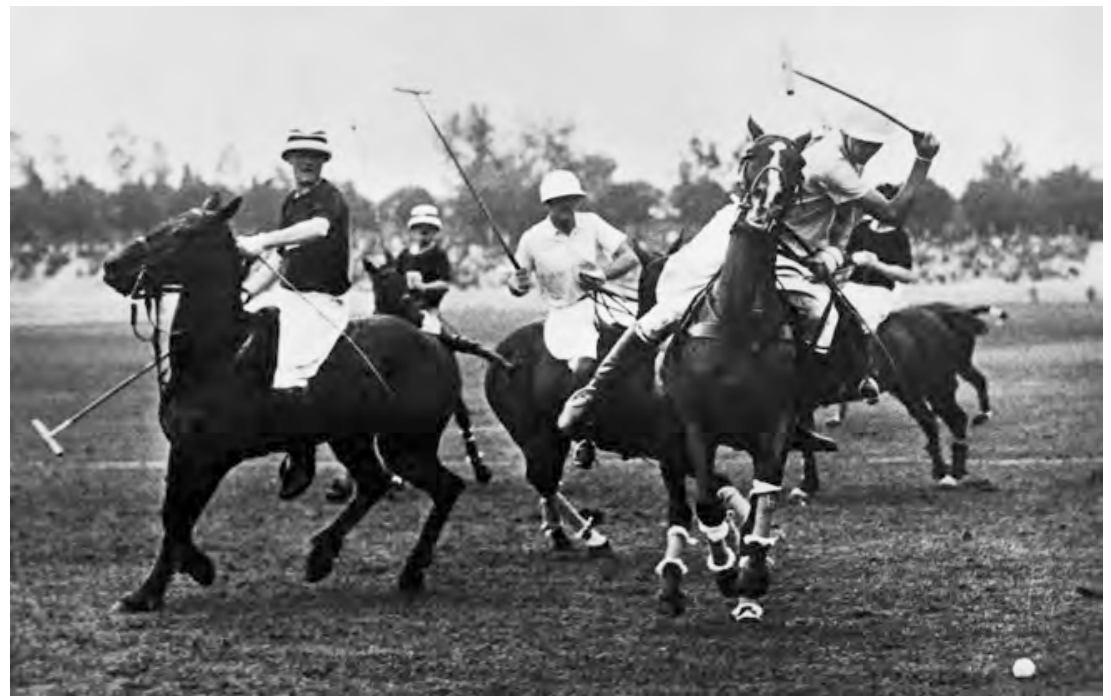

(Paul Wolff/Archiv Volker Kluge)

11 Die Fußballer, die sich den Amateurregeln angepasst hatten, kehrten 1936 zurück, ebenso Polo, das letztmals 1924 ausgetragen worden war. Neu im Programm waren Kanurennsport, Basketball und (Feld-)Handball. 
Brundage-Vorschlag: Hallen-Wettkämpfe im Winter, Frauen sollten unter sich bleiben

Bald nach dem Zweiten Weltkrieg, der erneut Deutschland und diesmal auch Japan „auf der Strafbank sah“, wurde die Programmdiskussion wieder aufgenommen. Edström, der 1946 die Leitung des IOC übernommen hatte, besaß als ehemaliger IAAF-Präsident die Weitsicht, den Gedankenaustausch gemeinsam mit den Fachverbänden zu führen. Man bildete eine gemischte Kommission, die von dem US-Amerikaner Avery Brundage, später von dem Finnen Erik von Frenckell geführt wurde.

Das letzte Wort hatte freilich das IOC, das auf seiner Session von 1946 zur „Zweiklassengesellschaft“ - obligatorisch/fakultativ - zurückkehrte. Die Entstehung neuer unabhängiger Staaten, die eigene NOKs gründeten, wurde zu einer Herausforderung. Hinzu kam der Beginn des Fernsehzeitalters, womit das olympische Interesse wuchs, aber auch die Begehrlichkeiten zunahmen: Die Wiederaufnahme von Bogenschießen, Golf, Rugby und Polo wurde beantragt, doch vom IOC genauso abgelehnt wie von Baseball, Schach, Rollsport, Judo, Volleyball und Tischtennis. Keine dieser Sportarten erhielt die für eine Regeländerung notwendige ZweidrittelMehrheit.

Widerstand gab es weiterhin gegen den Sport der Frauen. Es galt schon als Erfolg, dass der elitäre Herrenzirkel ihnen die Teilnahme in der Leichtathletik, Fechten, Turnen (1952 in Helsinki erstmals auch im Achtkampf und in der Gruppengymnastik), Schwimmen, Kanurennsport, Eiskunstlauf, Skisport, Segeln und an den letztmals 1948 ausgetragenen Kunstwettbewerben gestattete (CIO, 1949). ${ }^{12}$ Zusätzliche Wettbewerbe, etwa im Rudern oder Basketball, wie sie von der Sowjetunion, die 1952 die olympische Bühne betreten hatte, gefordert wurden, hatten vorerst keine Chance (IOC, 1955a).

Manches entbehrte jeder Logik: So plädierte das IOC 1939 mit 16:11Stimmen für Frauen-Eisschnelllauf bei den Winterspielen von 1940 in Garmisch-Partenkirchen, die wenig später wegen des Krieges abgesagt wurden. Als 1955 die Austragung dieser Disziplinen erneut debattiert wurde, erhielt der Antrag nur vier Ja-Stimmen. Ein Jahr verging, und siehe

12 Erstmals gestattete der internationale Verband (FIE) den Frauen 1952 in Helsinki die Teilnahme, nachdem er Ende 1948 die antiquierte „Herrenreiter“-Regel abgeschafft hatte. 
da! Nun wurden die Eisschnellläuferinnen doch für würdig befunden, 1960 in Squaw Valley an den Start zu gehen.

$\mathrm{Zu}$ den Dauerthemen jener Zeit gehörte die Befürchtung, dass die Sommerspiele mit rund 70 Ländern und nahezu 5000 Sportlern zu groß werden könnten. Brundage, der Edström 1952 im Präsidentenamt abgelöst hatte, sah die Lösung des Problems in der Eliminierung einiger fakultativer Sportarten und in der Auslagerung von Hallenwettkämpfen wie Boxen, Gewichtheben und Ringen in die Winterspiele. Neben allen Teamwettbewerben wollte er die Teilnahme der Frauen insgesamt rückgängig machen. Ihnen empfahl er: „... diese könnten ja wie in der Antike ihre eigenen Spiele austragen“ (Brundage, 1952).

Das war seinen Kollegen dann doch zu radikal. Baron von Frenckell als neuer Chef der Programmkommission plädierte 1953 dafür, nur noch solche Sportarten zuzulassen, die in 20 oder mehr Ländern praktiziert wurden. In den überarbeiteten Regeln von 1958 wurde jedoch die bisherige Klassifizierung fallengelassen. Dafür reduzierte man das Programm auf „mindestens 15 Sportarten“, die - als Minimum - in 25 Ländern ausgeübt werden sollten (IOC, 1958b, S. 20.). Es gab verschiedene Rechenmodelle, wie Teilnehmerzahlen von höchstens 5000 bis 6000 auch zukünftig zu erreichen wären (IOC, 1958a).

Ein erster Schritt war es, in den Mannschaftssportarten höchstens 16 Teams zuzulassen. Dem wachsenden Interesse an Fußball und Basketball kamen die verantwortlichen Verbände mit Qualifikationsturnieren entgegen. Die IAAF beschloss 1959 erstmals für die Olympischen Spiele in Rom 1960 Normen, die in einem feststehenden Zeitraum zu erfüllen waren. ${ }^{13}$

Dem wollte sich der Internationale Schwimmverband (FINA) keinesfalls anschließen. Er gab sich für 1960 mit zwei Startplätzen pro Land und Einzeldisziplin zufrieden, um im gleichen Atemzug mit der Einführung von Weltmeisterschaften zu drohen, worauf das IOC für 1964 drei neue Wettbewerbe und für 1968 weitere elf genehmigte. Die Welttitelkämpfe kamen 1973 dennoch. Erst 1976 folgte die FINA dem Beispiel der Leichtathletik und führte Qualifikationsnormen ein.

Es dauerte noch lange, ehe sich das IOC vom alten Denken einer Zeit verabschiedet hatte, in der interkontinentale Flugreisen und TV-Direkt-

13 Ein Startplatz pro Landesverband und Disziplin war garantiert. Wenn ein zweiter oder dritter Teilnehmer nominiert werden sollte, war die Erfüllung einer Olympianorm nachzuweisen. Die Regel galt nicht für Marathonlauf, Gehen und die Staffeln. 
übertragungen als Außergewöhnlichkeit wahrgenommen wurden. Mit dem Zerfall der großen Kolonialreiche war auch die olympische Welt größer geworden. Einige Zahlen: 1950 existierten 67 NOKs. Ein Jahrzehnt danach gab es bereits 92. Vor den Spielen von 1964 in Tokio hatte das IOC 117 Komitees anerkannt, davon 51 aus Afrika und Asien. Hingegen repräsentierten die 69 IOC-Mitglieder zum gleichen Zeitpunkt nur 53 Länder, von denen 38 zu Europa gehörten (Kluge, 1998).

Wie unterschiedlich selbst in diesem kleinen Kreis die Vorstellungen waren, wie ein modernes Programm aussehen sollte, zeigte sich 1961 bei einer IOC-Abstimmung. Von der Liste der möglichen 22 Sportarten, zu denen als Zugeständnis für die japanischen Gastgeber der nächsten Spiele auch Judo und Volleyball gehörten, erhielten nur sechs - Leichtathletik, Turnen, Rudern, Schwimmen, Gewichtheben und Ringen - die Zustimmung aller 43 Anwesenden. Hingegen fanden jeweils nur zwei „Olympier“, dass Fechten, Reiten und Judo unbedingt ausgetragen werden sollten. Und fürs Schießen blieb eine einzige Stimme übrig (IOC, 1961; IOC, 1962a). ${ }^{14}$

Als man zwei Jahre später erneut abstimmte, diesmal über die Spiele von Mexiko '68, für die das Programm auf 18 Sportarten reduziert werden sollte, ernteten allein die Leichtathletik und das Schwimmen die volle Stimmzahl. Verlierer des „Ausscheidungsrennens“ waren Judo, Handball, Bogenschießen und Volleyball (IOC, 1963). Die Volleyballer wurden 1965 aber doch noch zugelassen, als plötzlich ein Platz auf der Liste frei blieb. Denn Wasserball wurde nun unter dem Dach des Schwimmsports geführt.

Es mangelte also in den 1960er-Jahren nicht an Reformvorschlägen, von denen viele überfällig waren. Darunter fallen die Bemühungen, die „künstlichen“ Mannschaftswettkämpfe im Reiten, Modernen Fünfkampf und Turnen in eigenständige Disziplinen umzuwandeln, denn bis dahin war es üblich gewesen, deren Ergebnisse durch die Summierung der Einzelresultate zu ermitteln. Als das IOC entschied, ab 1964 die drei erstplatzierten Teams jeweils mit einer einzigen Medaille auszuzeichnen und deren Mitgliedern nur noch Diplome zu verleihen (IOC, 1962b), begannen einige Fachverbände, ihre Regeln zu ändern.

Der Internationale Turnverband (FIG) hatte schon 1960 Finalwettkämpfe für die besten Sechs an den Geräten eingeführt, und ab 1972 gab es auch separate Mehrkämpfe in der Mannschafts- und Einzelwertung. Die Reiter taten es ihnen nach. Lediglich in der strapaziösen Vielseitigkeitsprü-

14 Obwohl Handball und Bogenschießen 28 bzw. 26 Stimmen erhielten, wurden diese Sportarten weder 1964 noch 1968 ausgetragen. 
fung brauchte die FEI bis 1996, um sich für die Trennung in Einzel und Team zu entscheiden.

Im Modernen Fünfkampf handelte der Verband, dem von 1955 bis zur Trennung von 1993 auch Biathlon zugeordnet war, erst unter dem Druck des IOC und des Fernsehens. Nach dem Verzicht auf die Teamkonkurrenz wurde der UIPM 2000 in Sydney jedoch als Ausgleich ein Frauenwettbewerb zugestanden, der wie der der Männer an einem Tag über die Bühne zu gehen hatte.

\section{Mit Samaranch kam die finanzielle Blütezeit, die die olympische Familie wachsen ließ}

Mit Beginn der 1970er-Jahre bestand das offizielle Programm aus 21 Sportarten, die bei den nächsten vier Olympischen Spielen - 1972 bis 1984 ohne Abstriche durchgeführt wurden. Das IOC war zwar bereit, weitere IFs anzuerkennen, doch aus dem Status „Recognized International Federation" war nicht das Recht abzuleiten, dass deren Sport bei Olympischen Spielen ausgetragen würde. 1970 befanden sich zehn Fachverbände auf dieser "Warteliste“ (IOC, 1969).15 Aktuell sind es 42 - von American Football bis zur chinesischen Kampfsportart Wushu.

Die Zahl der Disziplinen bei Sommerspielen stieg deutlich an. Wurde 1968 noch in 173 Wettbewerben um Medaillen gestritten, so waren es 1972 bereits 195 und 1984 (insbesondere durch den Anstieg des weiblichen Anteils im Rudern, Handball, Basketball, Hockey, Synchronschwimmen und in der Rhythmischen Sportgymnastik) 221. Zwar gingen im gleichen Zeitraum die Teilnehmerzahlen teilweise deutlich zurück, aber nicht etwa durch neue Bescheidenheit, sondern sie war mit den Auswirkungen der politisch bedingten Boykotte von 1976 bis 1984 begründet.

Die Olympischen Spiele 1984, für die sich allein Los Angeles beworben hatte, waren die ersten, die vollkommen privat finanziert wurden. Sie wurden zu einem Wendepunkt der olympischen Geschichte, weil mit ihnen die globale Vermarktung begann. Mit dem 1980 gewählten IOCPräsidenten, dem Katalanen Juan Antonio Samaranch - von Beruf Bankkaufmann -, gelang es bereits im ersten Jahr, das IOC-Kapital von 385.000 Schweizer Franken auf 27,354 Millionen zu erhöhen. Von da an

15 Die Sportarten waren Flugsport, Baseball, Boules, Casting, Pelota, Rollerskating, Rugby, Tennis, Softball, Wasserski. 
stiegen die Gewinne permanent, und dank der Fernseheinnahmen wurde 1985 erstmals die 100-Millionen-Grenze überschritten.

Zusätzlich zum TV-Geschäft setzte Samaranch eine Kommission ein, die neue Finanzquellen erschließen sollte. Rückwirkend erfuhr das IOC auf der Session von 1983, dass er nach geheimen Gesprächen mit der in der Schweiz ansässigen und von Adidas-Chef Horst Dassler gegründeten Agentur „International Sports and Leisure“ (ISL) einen Vertrag ausgehandelt hatte, der den NOKs unerwartet Finanzmittel in die Hand gab, vorausgesetzt, dass sie ihre Vermarktungsrechte exklusiv an ISL abtraten.

Da das IOC für Los Angeles erstmals auch die Reisekosten für vier Sportler und zwei Funktionäre pro Land übernahm, entstanden neue NOKs, die sich von da an auch bei den Spielen mit Mini-Delegationen beteiligten. Gab es 1980 noch 145 NOKs, so waren es 1990 bereits 164 . Im Jahr 2000 existierten 200, gegenwärtig sind es 206 (Lyberg, 1996). Es lag an den Elementen, dass einer ähnlichen Entwicklung auf Eis und Schnee Grenzen gesetzt waren. Die Winterspiele dauerten seit 1924 üblicherweise zwischen zehn und zwölf Tage. Doch als das IOC 1986 einen neuen, ab 1994 gültigen Austragungszyklus einführte und die Dauer der Winterspiele auf 16 Tage verlängerte, um 1988 in Calgary dem USFernsehsender ABC zwei volle Wochenenden zur Vermarktung bieten zu können, langweilten sich viele Zuschauer angesichts eines Programms von nur 46 Entscheidungen und sehr viel mehr Sendezeit. Daran konnten auch die drei Demonstrationssportarten Freestyle, Shorttrack und Curling nichts ändern, die ab 1992 bzw. 1994 das offizielle Programm ergänzten.

Neue Disziplinen mussten her. Teilsportarten wie Langlauf, Nordische Kombination und Skispringen wurden eigenständig geführt und ihr Programm ausgebaut. Der Bob- und Schlittenverband (FIBT) erinnerte sich an das 1928 und 1948 in St. Moritz ausgetragene "olympische" Skeleton, das sich nicht nur auf dem Cresta Run in St. Moritz selbst, sondern ab Salt Lake City 2002 auch auf modernen Bahnen austragen ließ.

Anfangs gab es Bedenken, ob Snowboard, Freestyle oder Shorttrack schon genügend ausgereift wären, um mit den Traditionssportarten mithalten zu können, doch mit den Jahren schwanden die Vorbehalte. Was man bisweilen für kurzzeitige Jugendinteressen gehalten hatte, nahm bald einen festen Programmplatz ein. Den Zuschauern gefiel, was sie zu sehen bekamen.

Bestand der olympische Wintersport 1994 noch aus 61 Medaillenkonkurrenzen, so waren es 2002 bereits 78. Im Jahr 2018 wurde in PyeongChang die 100er-Grenze um zwei überschritten - und noch immer bietet das 16-Tage-Programm viel Raum. 
Anders sieht das bei den Sommerspielen aus. Zwar erhöhte das IOC mit den Jahren die Ansprüche für die Akzeptanz, doch proportional zur steigenden Bedeutung des Fernsehens wuchs das Bestreben der Interessenvertreter*innen, mit ihren Sportarten in die olympische Familie aufgenommen zu werden, zumal die TV-Gelder, die das IOC seit Mitte der 1960er-Jahre nach den Spielen ausschütten konnte, für manche überlebenswichtig wurden.

Verlangte das IOC 1972 von den Fachverbänden noch den Nachweis, dass ihr Sport mindestens in 25 Ländern und auf drei Kontinenten praktiziert wurde, so lautete das Minimum 1979 bereits 40 Länder/drei Kontinente. Nach Einführung des IOC-Entwicklungsprogramms „Olympic Solidarity“, mit dem die „Dritte-Welt“-Länder eine wirkliche Perspektive erhielten, wurde das Limit 1983 zuerst auf 50/3 und dann 1994 auf 75/4 erhöht. Nur Frauen und Wintersportler erhielten weiterhin Rabatt. ${ }^{16}$

Mit der Wahl von Samaranch begann eine Blütezeit für das IOC, das vorher bisweilen in Schwierigkeiten war, seinen finanziellen Verbindlichkeiten nachzukommen. Auch an Bewerberstädten für Olympische Spiele war fortan kein Mangel. Zudem entwickelte der Präsident einen Expansionsinstinkt, mit dem er die in Westeuropa zunehmende Skepsis gegenüber der Olympischen Bewegung durch eine Hinwendung nach Asien kompensieren konnte. Für Seoul 1988 wurde Tischtennis aufgenommen, und Tennis erlebte mit einem kontrovers diskutierten Beschluss auf der Session von 1987 sein Revival. Mit der als „Experiment“ deklarierten Zulassung der Profis waren die „offenen Spiele“, für die man 1981 auf dem Olympischen Kongress in Baden-Baden die Grundlagen gelegt hatte, Realität geworden. ${ }^{17}$

Im Wartemodus von Demonstrationssportarten bzw. Vorführungen verharrten 1988 Baseball, Frauen-Judo und Badminton, deren offizielles Debut dann vier Jahre später in Barcelona folgte. Softball, die weibliche Variante von Baseball, schloss sich 1996 an. Nur die Taekwondo-Kämpfer,

16 Für Frauendisziplinen und Wintersport galten folgende Limits: ab 1972 jeweils 25 Länder/2 Kontinente. Ab 1983: 35 bzw. 25/3, 1989: 35/3, 1994: 40/3. Für den Wintersport waren 25 Länder und drei Kontinente notwendig.

17 Nachdem Tennis 1964 und 1984 als Demonstration ausgetragen worden war, köderte die ITF 1981 die IOC-Mitglieder mit dem Versprechen, nur Amateure an den Start zu bringen. Tatsächlich waren es 1988 Profis, die sich verpflichten mussten, ihre Verträge für die Dauer der Spiele ruhen zu lassen und den von den NOKs nominierten Mannschaften anzugehören. Weitere Einschränkungen: Zugelassen waren nur die Jahrgänge 1964 und jünger. Die 64 Herren und 32 Damen mussten sich zudem mit ihrer Teilnahme am Davis bzw. Federation Cup qualifizieren. 
die sich ebenfalls im Beiprogramm von Seoul präsentieren konnten, mussten sich noch bis Sydney 2000 gedulden (Gueorguiey, 1996).

Bedenkt man, dass weltweit schätzungsweise mehr 600 Sportarten betrieben werden, von denen gegenwärtig 95 (+ 20 assoziierte) in der Global Association of International Sports Federations (früher SportAccord) organisiert sind, so erhebt sich spätestens hier die Frage, nach welchen Kriterien eigentlich die olympischen Sportarten ausgewählt wurden und was gegen die Anerkennung anderer sprach?

Neben gewachsenen Traditionen handelt es sich um Maßstäbe, die in Jahrzehnten durch die Olympische Programmkommission auf Grundlage intensiver Recherchen erarbeitet und die als Empfehlungen dem IOC-Exekutivkomitee vorgelegt wurden. Seit den 1970er-Jahren waren die Kriterien auch in der Olympischen Charta nachzulesen.

Nach den damaligen Regeln kam nur eine Amateursportart in die engere Wahl, und falls die zuständige Internationale Föderation über keinen Amateurbereich verfügte, so verlangte das IOC die Gründung eines Fachverbandes für olympischen Sport, wie das im Falle des Radsports geschah. 
Abbildung 4: Um Olympiamedaillen im Sportklettern wird erstmals bei den Spielen Tokio 2020 gekämpft. Verlangt wird die Kombination, die aus den Disziplinen Speedklettern, Bouldern (Klettern obne Seil) und Schwierigkeitsklettern besteht. Der Japaner Keita Dohi stellte sein Können bereits bei den Olympischen Jugendspielen 2018 in Buenos Aires unter Beweis.

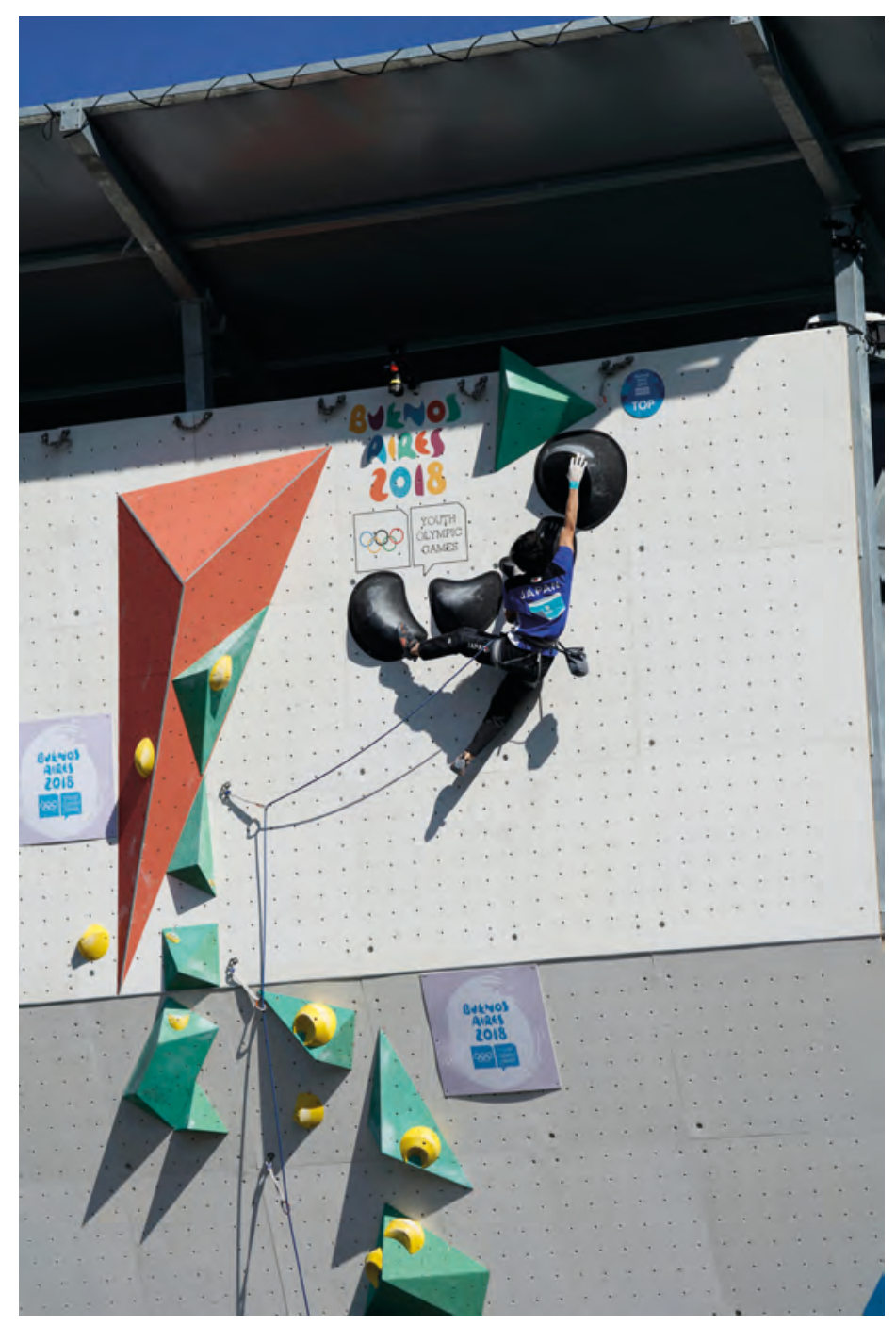

(picture-alliance) 
Gute Chancen, um die engere Wahl zu kommen, hatten nach den damaligen IOC-Kriterien Sportarten, die „einen starken Anteil an physischer Aktivität erfordern“ und „in denen Leistungen mit einem Minimum an menschlichem Irrtum gemessen werden können“. Bevorzugt gefördert wurden zudem Sportarten, deren Ausrüstung und Organisation keine hohen Ausgaben erfordern, während man solche mit einem Equipment, das nicht allen zur Verfügung stand, von vornherein ablehnte (IOC, 1978). Als auch der Motorsport Gefallen an den Olympischen Spielen fand, ergänzte das IOC die spätere Regel 45 um den Passus: „5. Sportarten, die von mechanischem Antrieb abhängig sind, werden nicht zugelassen“ (IOC, 1980).

\section{Alle Sportarten im Programm müssen sich einer regelmäßigen Revision unterziehen}

Spätestens zur Jahrtausendwende wurde klar, dass man auf Dauer die Olympische Bewegung nicht wie ein "Sonnengott" leiten konnte. Der mit den Olympischen Winterspielen in Salt Lake City verbundene Bestechungsskandal stürzte das IOC 1999 in seine tiefste Krise.

Auch die immer mehr ausufernden Dimensionen der Spiele waren besorgniserregend. Auf der Session 2001 in Moskau konfrontierte der frühere IOC-Vizepräsident Dick Pound seine Kollegen mit den Fakten. So war die Zahl der akkreditierten Personen bei den Sommerspielen von 1988 bis 2000 von 130.000 auf 200.000 gestiegen, die bei Winterspielen von 39.000 auf 90.000. Davon waren $72 \%$ Arbeitskräfte, neun Prozent Athlet*innen und Trainer*innen, acht Prozent Medienvertreter (IOC, 2002).

Die beim Amtsantritt von Samaranchs Nachfolger Jacques Rogge angekündigte Programmreform, die das Ziel hatte, sich mehr Spielraum zu verschaffen, scheiterte jedoch bereits im ersten Anlauf. Gegen die Streichung von drei Sportarten - Softball, Baseball und Moderner Fünfkampf - sprachen vor allem juristische Gründe, da ein Ausschluss laut Charta mit Blick auf Peking 2008 zuletzt sieben Jahren vorher möglich gewesen wäre (IOC, 2001). Die Entscheidung wurde deshalb auf die Zeit nach Athen 2004 vertagt.

Die nächsten drei Jahre wurden von der von Franco Carraro (Italien) geleiteten Programmkommission genutzt, um jede Sportart unter Einbeziehung von Experten nach 33 quantitativen und qualitativen Kriterien gründlich zu evaluieren. Bewertet wurden Informationen über die Verbreitung, Popularität und Entwicklung der Sportarten, aber auch 
Daten über TV-Sendezeiten, Einschaltquoten, Organisationskosten und „Athlete Welfare“, d.h. über den Stand des Antidopings.

Die Ergebnisse waren eindeutig: In der Universalität waren Leichtathletik (211 nationale Verbände) und Fußball (205) unschlagbar. Beide Sportarten - vor allem die Leichtathletik mit ihren damals 46 Disziplinen - hatten in Sydney und Athen auch den größten Anteil am Ticketverkauf, und sie besaßen das größte Werbepotenzial (Olympic Programme Commission, 2005). Als Nachteil für „König Fußball“ blieb bis heute, dass die FIFA nicht die besten Spieler*innen aufbieten will, weil sie den Wert ihrer Weltmeisterschaften keinesfalls schmälern möchte. Damit haftet dem olympischen Fußballturnier der Männer der Makel einer U23-WM an, weil die FIFA 1982 nach der Liberalisierung der Zulassungsregeln die Teilnahme auf diese Altersgruppe begrenzte.

Es war das demokratische Recht aller 105 IOC-Mitglieder, 2005 in Singapur über den Verbleib jeder einzelnen Sportart im Programm für London 2012 abzustimmen. Nur zwei - Baseball und Softball - erhielten nicht die erforderliche einfache Mehrheit (IOC, 2005). Hingegen verfügte die UIPM mit Sitz in Monaco über einflussreiche Fürsprecher. Ehrenpräsident Fürst Albert II. und Juan Antonio Samaranch Jr., 1. UIPM-Vizepräsident, machten sich für das Pentathlon stark. Vielleicht wirkte auch das Argument, dass der Moderne Fünfkampf Coubertins einziger Beitrag zum Sportprogramm gewesen war.

Dass erstmals seit 40 Jahren, seit dem kurzzeitigen Ausschluss von Judo, Sportarten gestrichen wurden, war ungewöhnlich, hatte aber Gründe: Softball mangelte es außerhalb von Nordamerika und Asien an Popularität. Das Interesse bei den Spielen in Sydney und Athen war gering, allerdings auch der Aufwand für den Bau einer 8000-Zuschauer-Arena. Die Kritik am Baseball konzentrierte sich auf die Tatsache, dass auch hier die Besten fehlten. Denen hatten die Besitzer der Profi-Ligen die Teilnahme untersagt. Eine Kampagne sollte es richten. Im Jahr 2012 fusionierten die beiden Verbände IBAF und ISF zur World Baseball Softball Confederation (WBSC) in der Hoffnung, 2020 in Tokio wieder dabei zu sein. Sie hatten richtig kalkuliert.

Auch Traditionssportarten wie Ringen mussten es akzeptieren, dass sich alle 28 IFs ausnahmslos jeweils nach den letzten Spielen einer kritischen Revision zu stellen haben. Grundlage war die 2007 veränderte Regel 46, nach der das IOC-Exekutivkomitee der IOC-Session ein Programm von mindestens 25 Sportarten vorschlug, die als Kern bezeichnet wurden und über die die IOC-Session en bloc abzustimmen hatte. Dem Organisationskomitee der nächsten Spiele wurde zudem das Recht zugestanden, zusätzliche Sportarten anzubieten, deren Austragung ebenfalls von der IOC- 
Session zu genehmigen war. Die Gesamtzahl der Sportarten einschließlich Kern sollte jedoch die Zahl 28 nicht übersteigen (IOC, 2007).

Als der Ringkampf von der IOC-Exekutive im Februar 2013 zusammen mit sieben weiteren Sportarten - Baseball/Softball, Karate, Rollsport, Sportklettern, Squash, Wakeboard und Wushu - auf eine Shortliste gesetzt wurde, ging ein Aufschrei durch die Medien, von denen sich allerdings bis dahin nur wenige gerade für „Randsportarten“ erwärmen konnten. Am vernünftigsten reagierten die Betroffenen selbst. Im Weltverband kam es zu einem Präsidentenwechsel. Um das Ringen attraktiver und spannender zu gestalten, wurden die seit 1975 ständig veränderten Regeln erneut reformiert, außerdem der Anteil der Frauen auf und neben der Matte vergrößert.

Das IOC honorierte diese Anstrengungen. Im September 2013 wurde der FILA (heute United World Wrestling/UWW) mit der knappen Mehrheit von einer Stimme - nötig waren 49 - für 2020 und 2024 Bewährung zugebilligt, während erneut Baseball/Softball (24) und diesmal auch Squash (22) durchfielen (IOC, 2013).

Der neue Trend: Die Spiele werden jünger, bunter und weiblicher, aber nicht größer

Mit der 2014 beschlossenen Olympic Agenda 2020, die die Handschrift des amtierenden IOC-Präsidenten Thomas Bach trägt und einstimmig vom IOC angenommen wurde, erhielt die Olympische Bewegung eine neue strategische Ausrichtung. Drei der 40 vom Exekutivkomitee ausgearbeiteten Empfehlungen betrafen das Programm, das „from a sport-based to an event-based programme" umgewandelt wurde. Mit anderen Worten: Statt auf der Zahl 28 zu beharren, wurde die Obergrenze der Disziplinen (events) auf 310 (Sommer) bzw. 100 (im Winter) festgeschrieben (IOC, 2015).

In Rio de Janeiro 2016 wurde diese Vorgabe eingehalten. Mit je zweimal Golf und Siebener-Rugby erhöhte sich die Zahl der Medaillenwettbewerbe gegenüber London auf 306. Anders bei der Gesamtteilnehmerzahl, die mit 11.238 deutlich über dem Limit von 10.500 lag.

Damit nicht genug. Da das IOC den Olympia-Gastgebern die Möglichkeit ließ, mit zusätzlichen Sportarten das Programm auf eigene Kosten zu ergänzen, griff Tokio 2020 mit vollen Händen zu. Auf der Session in Rio kehrte nicht nur Baseball als Japans Nationalsport Nr. 1 zurück, sondern auch Softball. Hinzu kamen als neue Sportarten Karate, Skateboard, Sportklettern und Surfen, die sich bei den Olympischen Jugendspielen, den 
Youth Olympic Games (YOG), dem ,juvenilen Versuchslabor“, empfohlen hatten (IOC, 2016).

Was dem Einen recht ist, sollte dem Anderen jedoch nicht billig sein - erst recht nicht in Corona-Zeiten. Im Programm von Paris 2024 stehen alle Sportarten der 28 anerkannten IFs. Hinzu kommen wie in Tokio 2020 Skateboard, Sportklettern und Surfen sowie als einziger neuer Sport das zur World Dance Sports Federation zählende Breaking (Breakdancing). Die Zahl der Medaillenwettbewerbe will Paris 2024 gegenüber Tokio 2020 um zehn auf 329 reduzieren, ebenso die Gesamtzahl der Athlet"innen auf längst festgelegte 10.500 (IOC, 2019).

Abbildung 5: Der akrobatische Breakdance, der einst auf den Straßen von New York als Hip-Hop Lifestyle entstand, wird 2024 in Paris seine olympische Premiere erleben. Die Hoffnungen des Gastgebers könnten dann auf „B-Boy" Martin Lejeune ruhen, der bei den Olympischen Jugendspielen 2018 in der Kategorie Breaking eine Silbermedaille gewann.

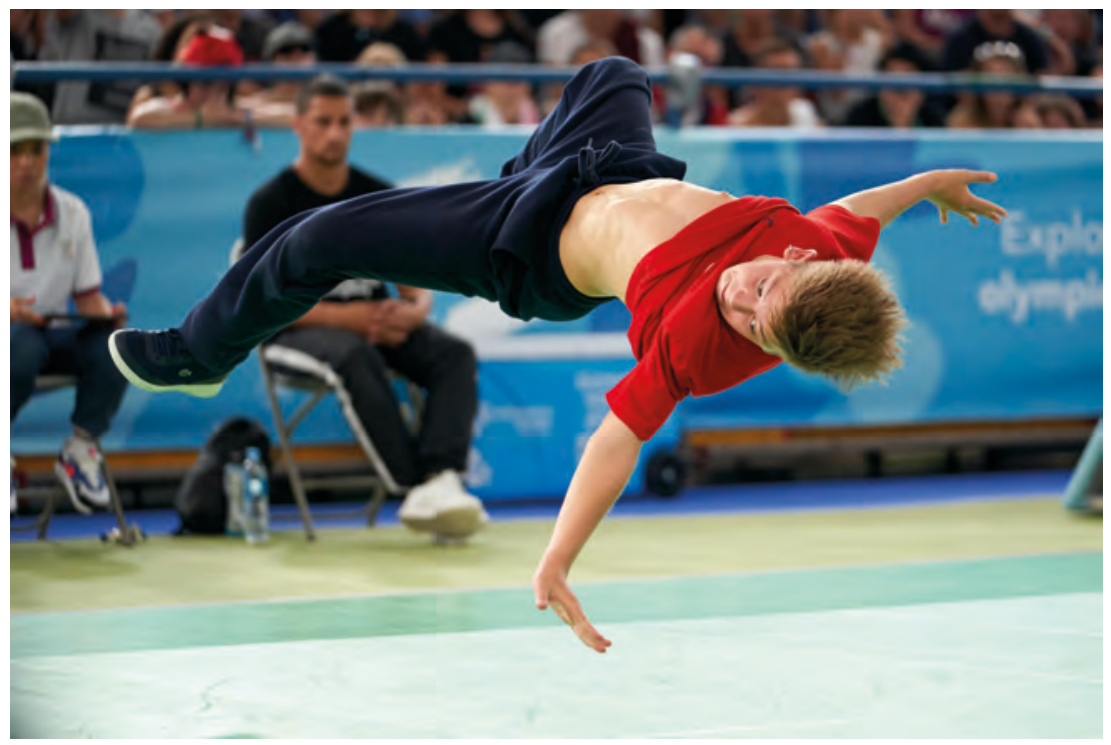

(picture-alliance)

Für manche Sportarten heißt das, auch Opfer zu bringen, was nicht immer die Falschen trifft. Das permanent vom Doping gebeutelte Gewichtheben wird auf je fünf Klassen für Männer und Frauen reduziert, die Boxer, 
deren Dachverband (AIBA) 2019 wegen langjähriger Unregelmäßigkeiten vom IOC suspendiert wurde, auf sieben bzw. sechs. Zu den Gewinnern gehören die Frauen, die zum ersten Mal mit 50 Prozent der Disziplinen die volle Geschlechtergleichberechtigung erleben werden. ${ }^{18}$ Was für eine Entwicklung! In Paris 1900 waren es nur 22. Wie sich eines Tages die aktuell diskutierte Genderproblematik auswirken wird, lässt sich heute noch nicht voraussagen. Die Zunahme von Mixed-Wettbewerben deutet zumindest an, dass es bei der klassischen Geschlechter-Einteilung nicht bleiben muss.

Dass die olympische Welt globaler und vielfältiger geworden ist, beweisen die vielen neuen Sportarten, vor allem solche, die in Asien große Popularität genießen. Begünstigt wurden die Anerkennung von Judo, Volleyball, Taekwondo, Karate oder Bogenschießen aber auch durch die Tatsache, dass Japan, Südkorea und die Volksrepublik China bereits mehrfach olympische Gastgeber wurden. Nicht zu vergessen die Interessen der Sportartikelindustrie und anderer Stakeholder, die mit Hilfe der Olympischen Spiele für sich neue Märkte erschlossen haben.

Mit der Olympic Agenda 2020 und ihrer Fortsetzung als Agenda 20+5 fand das IOC kreative Lösungen, damit die Olympischen Spiele auch in Zukunft organisierbar und finanzierbar bleiben. Die Interessen junger Generationen werden im Programm ebenso berücksichtigt, ohne aber die DNA der Spiele zu beschädigen. Die Olympischen Spiele von Paris 2024 sollen zeigen, dass es möglich ist, das ständige Wachstum zu bremsen und die Dimensionen zu reduzieren.

\section{Literatur:}

Brundage, A. (1952). Circular letter. Olympic Studies Centre (OSC).

Czechoslovak Organising Committee (Hrsg.) (1925). Minutes of the Technical Olympic Congress, S. 51-52. Prag.

CIO (1949). Règles Olympiques, Regel 41, S. 18. Lausanne: IOC.

Coubertin, P. de, Philemon, T. J., Politis, N. G., \& Anninos, C. (1897). Die Olympischen Spiele 776 v. Chr.-1896 n. Chr. (Band 2). Athen: Carl Beck.

Coubertin, P. de (1912). Les femmes aux Jeux Olympiques. Revue Olympique, 109111.

18 Bei den Spielen in Tokio wurden 48,8 Prozent erreicht; in Rio 2016 waren es noch 45,6\%. Die Zahl der gemischten Wettbewerbe in Paris wird von 18 (Tokio) auf 22 steigen. 
Coubertin, P. de (1914). La décadence des sports d'hiver. Revue Olympique, 99, 39f.

Coubertin, P. de (1974). Einundzwanzig Jahre Sportkampagne (1887-1908). Ratingen: Henn Verlag.

Coubertin, P. de (1997). Olympic Memoirs. Lausanne: IOC.

Durry, J. (2019). The 'Coubertin Project' Paris 1900: What Might Have Been. Journal of Olympic History, 27(3), 8-19.

Gueorguiev, N. (1996). Analysis of the Olympic Programme 1896-1995. Lausanne: IOC.

IOC (1894a). Bulletin du Comité International, 1, S. 2-4.

IOC (1894b). Les Jeux Olympiques dans le passé et dans l'avenir. Bulletin du Comité International, 2, S. 3-4.

IOC (1894c). Protokoll. Olympischer Kongress Paris, Kommission für Olympische Spiele, 19. Juni 1894, S. 3-9. Lausanne: Olympic Studies Centre (OSC).

IOC (1919). Congrès des Comités Olympiques Nationaux tenu à Paris en Juin 1914. Lausanne: OSC.

IOC (1921). Congrès Olympique de Lausanne, 2-7 Juin 1921. Paris: Barré.

IOC (1930). Minutes of the Olympic Congress of Berlin 1930, S. 25.

IOC (1955a). Extract from the Minutes, Konferenz IOC-Exekutive und Ifs. Bulletin du Comité International Olympique, 52, S. 15.

IOC (1955b). Olympische Charta 1955, Regel 27. S. 14. Lausanne: IOC.

IOC (1958a). Zirkularbrief an alle IFs. Lausanne : Olympic Studies Centre (OSC).

IOC (1958b). Olympische Charta 1958, Regel 30, S. 20. Lausanne: IOC.

IOC (1961). Protokoll, 59. IOC Session, Athen 1961, S. 12-13. Lausanne: Olympic Studies Centre (OSC).

IOC (1962a). Olympische Charta 1962, Regel 30, S. 20. Lausanne: IOC.

IOC (1962b). Olympische Charta 1962, Regel 41, S. 26. Lausanne: IOC.

IOC (1963). Protokoll, 61. IOC Session, Baden-Baden 1963, S. 4, Annex No. 2. Lausanne: Olympic Studies Centre (OSC).

IOC (1969). Olympic Directory 1969, S. 50-51.

IOC (1978). Olympische Charta 1978 (provisional edition), By-laws VIII - To Rule 29, $S$ 49. Lausanne: IOC.

IOC (1980). Olympische Charta 1980 (provisional edition), Rule 45, S 48. Lausanne: IOC.

IOC (2001). Olympische Charta 2001, Regel 52, 1.1.4. S. 75. Lausanne: IOC.

IOC (2002). Protokoll, 114. IOC-Session, Mexiko-Stadt 2002, S. 14. Lausanne: Olympic Studies Centre (OSC).

IOC (2005). Protokoll, 117. IOC-Session, Singapore 2005, S. 21-22. Lausanne: Olympic Studies Centre (OSC).

IOC (2007). Olympische Charta 2007, Regel 46, By-Law 1.1.4. S. 87. Lausanne: IOC.

IOC (2013). Protokoll. 125. IOC-Session, Buenos-Aires 2013, S. 29. Lausanne: Olympic Studies Centre (OSC). 
IOC (2015). Olympische Charta 2015, Regel 45, By-Law 3.3.2. S. 87, Lausanne: IOC.

IOC (2016). Protokoll, 129. IOC-Session, Rio de Janeiro 2016, S. 39-41. Lausanne: Olympic Studies Centre (OSC).

IOC (2019). Protokoll, 134. IOC-Session, Lausanne 2019, S.71-75. Lausanne: Olympic Studies Centre (OSC).

IOC (2017). Directory, S. 162-163.

Kluge, V. (1998). Olympische Sommerspiele. Die Chronik II, London 1948 - Tokio 1964. Berlin: Sportverlag.

Kluge, V. (2019), The Rebels of 1894 and a Visionary Activist- Journal of Olympic History, 27 (1). S. 4-19.

Lucas, C. J. P. (1905). The Olympic Games 1904. S. 143. St. Louis: Woodard \& Tiernan.

Lyberg, W. (1996). Fabulous 100 Years of the IOC. Facts - Figures - and Much, Much More. IOC.

Mallon, B., \& Widlund, T. (1998). The 1896 Olympic Games. Results for All Competitors in All Events, with Commentary. Jefferson, North Carolina: McFarland.

Müller, N. (1981). Von Paris bis Baden-Baden. Die Olympischen Kongresse 1894-1981. Niedernhausen: Schors-Verlag.

Olympic Programme Commission (2005). Report to the 117th IOC Session. Singapore.

Sullivan, J. E. (1905). Spalding's Official Atbletic Almanac for 1905, Olympic Games Number. New York: American Sports Publishing Co. 
